\title{
Eating disorders during COVID-19 pandemic: the experience of Italian healthcare providers
}

\author{
Georgia Colleluori $^{1,2} \oplus \cdot$ Ilaria Goria ${ }^{2} \cdot$ Claudia Zillanti $^{2} \cdot$ Simonetta Marucci ${ }^{2,3} \cdot$ Laura Dalla Ragione $^{2,3}$
}

Received: 25 October 2020 / Accepted: 13 January 2021 / Published online: 9 February 2021

(c) The Author(s), under exclusive licence to Springer Nature Switzerland AG part of Springer Nature 2021

\begin{abstract}
Purpose Due to COVID-19 pandemic, the Italian population lived in quarantine from March to May 2020 (lockdown phase I). Restrictions impacted individuals' psychological health, especially in those with eating disorders (ED). Healthcare providers (HCPs) treating ED provided assistance by telemedicine and/or in walk-in clinics. We hypothesize that social restrictions represented a great stressor for ED patients and HCPs, negatively impacted their therapeutic alliance, and affected the frequency of dysfunctional behaviors.

Methods This cross-sectional study consisted of an online survey investigating the experience of HCPs involved in ED treatment, with a specific focus on difficulties concerning the therapeutic efficacy. Questionnaire ( $n .18$ questions) was formulated ad hoc by our research team and sent by e-mail to Italian HCPs registered on online platforms. HCPs included ED experts specialized in psychology, nutrition or medicine. Data were collected during lockdown phase I and referred to patients with Anorexia Nervosa-(AN), Bulimia Nervosa (BN)—and Binge-Eating Disorder-(BED).

Results One-hundred questionnaires were collected; 84 and 76 were included in our qualitative and quantitative analyses, respectively. Thirty-six\% of HCPs felt their therapeutic intervention was unsuccessful, 37\% complained compromised therapeutic alliance. Changes in frequency of compensatory behaviors (increased in $41 \% \mathrm{AN}$ and 49,5\% BN; reduced in 14,6\% AN and $21,8 \% \mathrm{BN}$ ) and binge-eating episodes (increased in 53,3\% BN and 30,5\% BED; reduced in 30,7\% BN and 24,7\% BED) were experienced and ascribed to augmented patient's anxiety. Disorders switches and variation in dysfunctional conducts frequency were both significantly related to ED category ( $p<0.05$ for all). Concentration techniques were recognized as useful to offset such negative outcomes.

Conclusion According to HCPs, social restrictions affected the frequency of dysfunctional behaviors in ED patients and the efficacy of their therapeutic intervention. Further long-term studies are needed to confirm our data in a larger sample size. Level IV Novel results from a cross-sectional study.
\end{abstract}

Keywords Eating disorders $\cdot$ COVID-19 $\cdot$ Healthcare providers $\cdot$ Anorexia nervosa $\cdot$ Bulimia nervosa $\cdot$ Binge-eating disorder

\section{Introduction}

Georgia Colleluori, Ilaria Goria and Claudia Zillanti contributed equally

Georgia Colleluori

g.colleluori@pm.univpm.it

1 Department of Experimental and Clinical Medicine, Marche Polytechnic University, Via Tronto 10A, 60020 Ancona, Italy

2 Eating Disorders Services-USL N. 1 'Palazzo Francisci', Todi, Italy

3 Food Science and Human Nutrition Unit, University Campus Biomedico of Rome, Via Alvaro del Portillo, Rome, Italy
Since December 2019, the Severe Acute Respiratory Syndrome CoronaVirus 2 (SARS-CoV-2) has spread globally resulting in a worldwide health crisis which caused over one million deaths. From March the 9th to May the 18th 2020, the Italian government has imposed a state of national quarantine, a timeframe known as lockdown phase I. The lockdown phase I consisted of strict norms deeply limiting social interactions and confining the population into their dwellings except for very few concessions (e.g., grocery, urgent medical assistance). Restrictions deeply impacted individuals' psychological health [1], especially in those 
suffering from eating disorders (ED). Patients with ED are in fact considered highly fragile due to the associated psychiatric comorbidities and metabolic anomalies [2]. Such elevated vulnerability makes them particularly susceptible to stress [2,3]. The COVID-19 pandemic and the consequent lockdown have been a great element of stress which worsened dysfunctional behaviors among ED patients [3-5]. As reported by Termorshuizen and colleagues in a study conducted in the United States and Netherland, during the COVID-19 pandemic, restriction practices increased among patients with anorexia nervosa, while binge-eating episodes augmented among those with bulimia nervosa and binge eating disorders [4]. Similar data were also collected by Phillipou and colleagues [6]. The impossibility of going out, the lack of social interactions, and constrictions within the family environment were all elements of stress able to exacerbate ED symptomatology. In the context of COVID-19 pandemic, it is thus crucial to assess not only the efficacy of social restrictions in limiting the infection outbreak, but also their psychologic consequences, especially among people with psychologic illnesses such as ED [5].

Healthcare providers (HCPs) involved in the treatment of ED continued to provide assistance either by telemedicine or in walk-in clinics. However, in Italy the online assistance had never been used before and such inexperience could negatively affect the efficacy of HCPs intervention. On the other side, also the in-person visit could be less effective due to the fear of the infections, to the use of Personal Protection Equipment (PPE), and because of the required distancing and environment disinfection activities. Changes in the therapeutic setting and practices could be very challenging for HCPs. However, how the setting modification affected HCPs assistance to ED patients has not been explored. Importantly, HCPs may have also experienced personal difficulties due to the pandemic, similarly impacting the quality of their care $[5,7]$. Most of the studies regarding ED focus on patients, while HCPs experiences remain poorly investigated $[4,6]$. Considering the crucial role of HCPs in the treatment of $\mathrm{ED}$, the comprehension of the impact of the COVID-19 pandemic on their therapeutic experience is critical to identify obstacles and potential solutions to guarantee a proper level of care.

The purpose of this study was to assess whether social restrictions represented a stressor for ED patients and their HCPs, if they affected their therapeutic alliance, and altered the frequency of dysfunctional behaviors. In this study we investigated HCPs experience during the Italian lockdown phase I and the impact of the social restrictions and of the pandemic environment. Furthermore, based on HCPs experience, we aimed to identify useful strategies able to offset complications lived during such period.

\section{Methods}

\section{Questionnaire and targets}

In this cross-sectional study we performed an online survey to investigate the experience of HCPs involved in ED treatment, e.g., psychiatrists, psychotherapists, nutritionists, dietitians and educators (social workers). The questionnaire entitled "Eating Disorders at time of COVID-19", was formulated ad hoc by our research team and administered through the Google Form platform. It was sent by e-mail to Italian HCPs registered on online platforms dedicated to ED and belonging to either public or private health centers specialized in the treatment of such disorders. The survey was conducted nationwide and none of the Italian regions was excluded. Data were collected during the lockdown phase I (March 9th-May18th 2020) and HCPs were asked to refer only to what experienced during that timeframe when answering the questions. Furthermore, HCPs were asked to refer to patients suffering from Anorexia Nervosa and atypical anorexia nervosa (AN), Bulimia Nervosa and low frequency bulimia nervosa (BN), Binge Eating Disorder and low frequency binge eating disorder (BED), as defined by the DSMV classification [8] HCPs were asked to not refer to patients suffering from ED different from the above listed ones (e.g., night eating syndrome, vigorexia or ortorexia). Survey was completed anonymously. Our questionnaire can be found in the supplementary material.

The objective of the questionnaire was to assess the experience of HCPs concerning the therapeutic practice during the lockdown phase I. The questionnaire consisted of two macrosections, one investigating patients experience based on the HCPs and the second one centered on HCPs themselves. Each section included quantitative and qualitative questions. The main goal of the quantitative questions was to assess changes in the frequency of dysfunctional behaviors among patients compared to the pre-lockdown (e.g., bingeing and compensatory conducts) and how many HCPs experienced difficulties in the establishment of a therapeutic alliance with patients. For this section, HCPs were asked to report the actual number of patients fitting the investigated topic. On the other side, the main objective of the qualitative investigation was to identify what kind of difficulties the HCPs experienced and useful strategies able to counteract them. Qualitative questions were either formulated in the form of "opened questions" or in the "true/ false" format. Specifically, for the first set of questions (1-4), the thematic area was "type of therapy and patients" (e.g., expertise -nutrition vs psychology; type of visit -in person vs telemedicine; patients number and disorder types). The second thematic area (questions 5-12) was "dysfunctional behaviors" (e.g., variation in frequency of compensatory, 
bingeing or restriction conducts, disorders switches, symptoms worsening that required modification in the therapeutic strategy). Questions (13-14) theme was "patients experience according to HCPs" and explored patients' difficulties and useful strategies to counteract them. For the last set of questions (15-18), theme was "HCPs experience" investigating therapists' difficulties during their assistance. For further details concerning the questionnaire please see the supplementary material.

Questions investigating changes in the frequency of dysfunctional behaviors shared features in common with the study from Termoshuizen [4], since they were specifically evaluating variations in bingeing, compensatory or restriction conducts among patients. Furthermore, the same study also assessed patients anxiety levels and fear of being infected, of gaining weight or not being able to exercise. However, although these specific topics of investigation were similar between our study and the one of Termoshuizen, our survey is the first one reporting HCPs perspective and not patients' one, focused on the Italian population.

The low number of questions ( $n$. 18) was chosen to facilitate survey completion and to keep it within 10 minutes. Main outcomes of the first section were: changes in the frequency of bingeing and compensatory behaviors and number of switches episodes from a disorder to another among patients. Main outcome of the second section was the assessment of the number of HCPs experiencing difficulties in the creation and maintenance of a therapeutic alliance and identification of useful approaches to offset negative outcomes due to the pandemic.

\section{Data analysis}

All questionnaires whose quantitative answers were incoherent were excluded from the quantitative, but not from the qualitative, assessment. We excluded for example questionnaires from HCPs who stated to follow a total of $n$ patients with ED during the lockdown phase I, but whose summation of subjects suffering from each specific disorder $(\mathrm{AN}+\mathrm{BN}+\mathrm{BED})$ was greater than $n$. Data from the quantitative subsection referring to each ED category refers to the total number of subject suffering from the specific ED (prevalence \% of AN patients who increased compensatory behaviors on the total AN patients followed during the lockdown phase I). Between ED group comparison in changes in the frequency of dysfunctional behaviors were assessed by Chi-Square. Items investigated using "true/ false" questions are reported as prevalence of HCPs providing a positive answer on the total included in the analyses. HCPs answers to opened questions belonging to the qualitative sections were carefully read and, in case they shared common features (at least two similar answers), they were discussed among the results. Surveys from HCPs who did not respond to the qualitative question "Which difficulties in the relation with your patients did you experience during the lockdown phase I?" were not included in the qualitative analyses. Data were managed using Microsoft Excel v.16.43, while SPSS Statics v24 was used for data analyses; $p<0.05$ was considered statistical significant.

\section{Results}

Nine-hundred fifty-eight questionnaires were sent to HCPs nationwide. One-hundred surveys were collected and 76 were selected for the quantitative analyses, while 84 for the qualitative one based on the inclusion criteria defined in the methods section. HCPs who completed the questionnaire consisted of 43 psychologists/psychotherapists, 43 experts in nutrition (dietitians, nutritionists and MD specialized in nutrition), 13 psychiatrists and 1 educator.

\section{ED dysfunctional behaviors}

Answers included in the quantitative analyses were provided by 76 HCPs and referred to 453 patients suffering from ED. Among them, 439 were followed online by telemedicine, while 54 were met in person in walk-in clinics (Fig. 1a). One-hundred seventy-eight subjects suffered from AN, 101 from BN and 174 from BED (Fig. 1b). Switching episodes were observed as follows: $8,4 \%$ of $\mathrm{AN}(n=15)$ switched to $\mathrm{BN} ; 8,9(n=9)$ and $10(n=10)$ of $\mathrm{BN}$ switched to $\mathrm{AN}$ and $\mathrm{BED}$, respectively; $5,2 \%$ of $\mathrm{BED}(n=9)$ switched to $\mathrm{BN}$, while none of BED or AN switched into each other. Our analyses revealed a significant relation between switches episodes and eating disorder categories $(p=0.001)$, with a higher percentage of $\mathrm{BN}$ patients undergoing them. Fortytwo patients suffering from AN, 24 from BN and 27 from BED increased the frequency of the visits with their HCPs (Fig. 1c); however, there was no association between the increase in the visits frequency and ED category $(p=0.113)$. New patients starting the therapy for the first time were less than $10 \%$ of the total of subjects suffering from each specific disorder (Fig. 1c). Considering the known association between stress and dysfunctional behavior, we investigated changes in the frequency of such conducts. Figure 1 shows the number of patients who modified the frequency of dysfunctional behaviors such as binge-eating and compensatory conducts. According to therapists, a variation in the frequency of compensatory behaviors such as purging, vomiting, starvation and excess of exercise was observed in patients with AN (73 increased and 26 reduced) and BN (50 increased and 22 reduced) (Fig. 1d). These variations were significantly related to ED category $(p=0.029)$, with higher number of $\mathrm{BN}$ patients experiencing changes in their frequency during the lockdown (both directions, reduction 
A Type of Visit (\%)

B Type of Eating Disorders (\%)
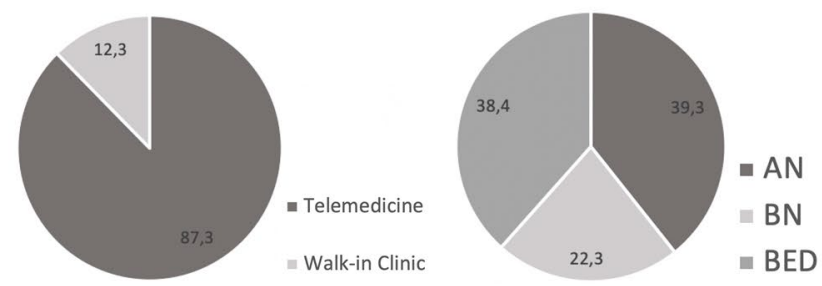

C Patients (\%) who started the therapy for the first time or who increased the number of visits

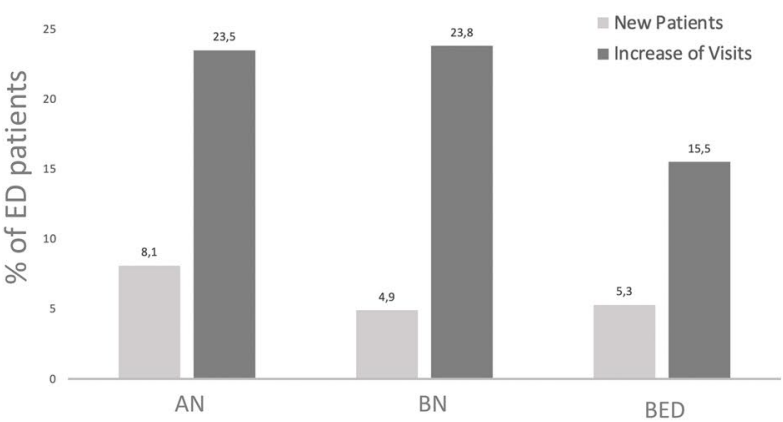

Fig. 1 Eating disorders during the lockdown due to COVID-19 pandemic according to HCPs. a Percentage of therapist performing in person visits (walk-in clinic) or telemedicine. b Type of eating disorder followed during the lockdown (\% value). c Percentage of patients initiating the therapy for the first time and percentage of patients who increased visits frequency according to each eating disorder category. Percentage of patients who variated the frequency of compensatory

and increase). Furthermore, bingeing episodes changed in weekly frequency among BED (53 increased and 43 reduced) and $\mathrm{BN}$ (increase 54 and 31 reduced) as showed in Fig. 1e. Our analyses revealed a significant relation between bingeing conducts and ED categories $(p<0.0001)$, with BN experiencing the highest variation. The high variability in the changes of the frequency of bingeing conducts within each ED categories could be due to the fact that such behaviors are usually performed in loneliness. Hence, the closed proximity to family members during the lockdown may have contributed to the reduction in conducts frequency among patients living in a shared environment. On the other side, social restrictions may have led to bingeing frequency increase in those living alone. Further studies should assess the characteristics of the subgroup of patients increasing the number of bingeing conducts to better comprehend such phenomenon. There was a significant relation with dysfunctional behaviors and ED category, with the highest number of $\mathrm{BN}$ experiencing variation, a finding that could be either related to the higher occurrence of switches among BN (which could explain for example the variation in compensatory conducts) or higher sufferance during the lockdown.
D Patients (\%) who changed the weekly frequency of compensatory conducts*
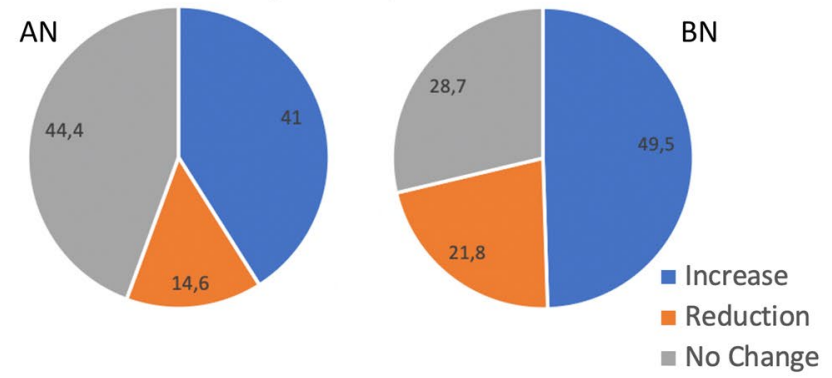

E Patients (\%) who changed the weekly frequency of bingeing conducts*
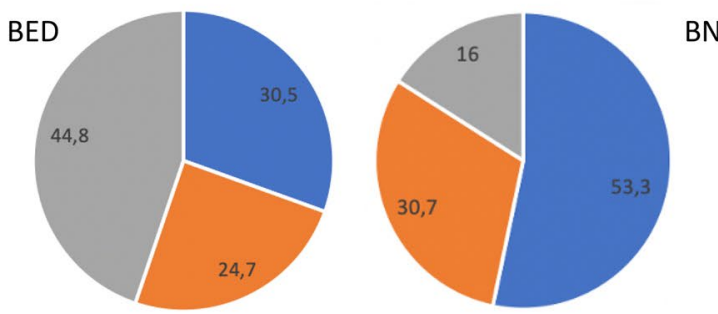

(d) and binge-eating (e) conducts according to each eating disorder category. $* p<0.05$ indicating a significant relation between variation in dysfunctional behavior frequency and ED category. $A N$ anorexia nervosa and atypical anorexia nervosa, $B N$ bulimia nervosa and lowfrequency bulimia nervosa, $B E D$ binge eating disorder and low-frequency binge eating disorder

Our data are in part consistent with the ones available in the literature, investigating the frequency of compensatory and bingeing behaviors among ED during the pandemic [4]. For most of ED patients, the pharmacotherapy was not modified as reported by the $75 \%$ of HCPs. This last data must be interpreted with caution since not all HCPs interviewed are allowed to prescribe drugs.

\section{Patients' experience according to HCPs}

The qualitative assessment had the objective to investigate the difficulties experienced by HCPs concerning ED patients management and to identify useful strategies able to counteract such problematics during the lockdown phase I. Data from the literature exploring the consequences of a pandemic state, evidenced symptoms worsening, increased anxiety levels and higher stress due to the risk of infection, all aspects accompanied by elevated dysfunctional thought on food and body image [9]. According to over $83 \%$ of HCPs, patients struggled in managing mood fluctuation, dysfunctional overthinking and anxiety (Fig. 2a). Furthermore, patients' discomforts were also ascribed to the isolation from their loved 
ones and condition of mourning or illness (Fig. 2a). On the other side, $34 \%$ of HCPs reported that their patients did not experience any difficulties (Fig. 2a). This report is consistent with the data from the literature reporting patients' struggles during periods of stress.

\section{HCPs experience}

Twenty-six\% of HCPs experienced personal difficulties that interfered with the therapeutic assistance during the lockdown phase I. However, the specifics of such personal discomfort were not further investigated to keep the questionnaire short and focused on therapeutic assistance and
ED patients. Among the difficulties experienced by HCPs there were: an elevated sense of therapeutic inefficacy, compromised therapeutic alliance and non-verbal communication especially through telemedicine as compared to the in-person visits, while the distraction due to the use of PPE was more prevalent among HCPs working in the walk-in clinic (Fig. 2b). Anxiety due to the fear of infection was similarly experienced in both assistance modalities (Fig. 2b). Importantly, 32\% of therapists $(n=27)$ reported elevated difficulties in the involvement of patients' family in the therapy. In general, based on the interviewed HCPs, both approaches, telemedicine and walk-in clinics, presented several challenges.

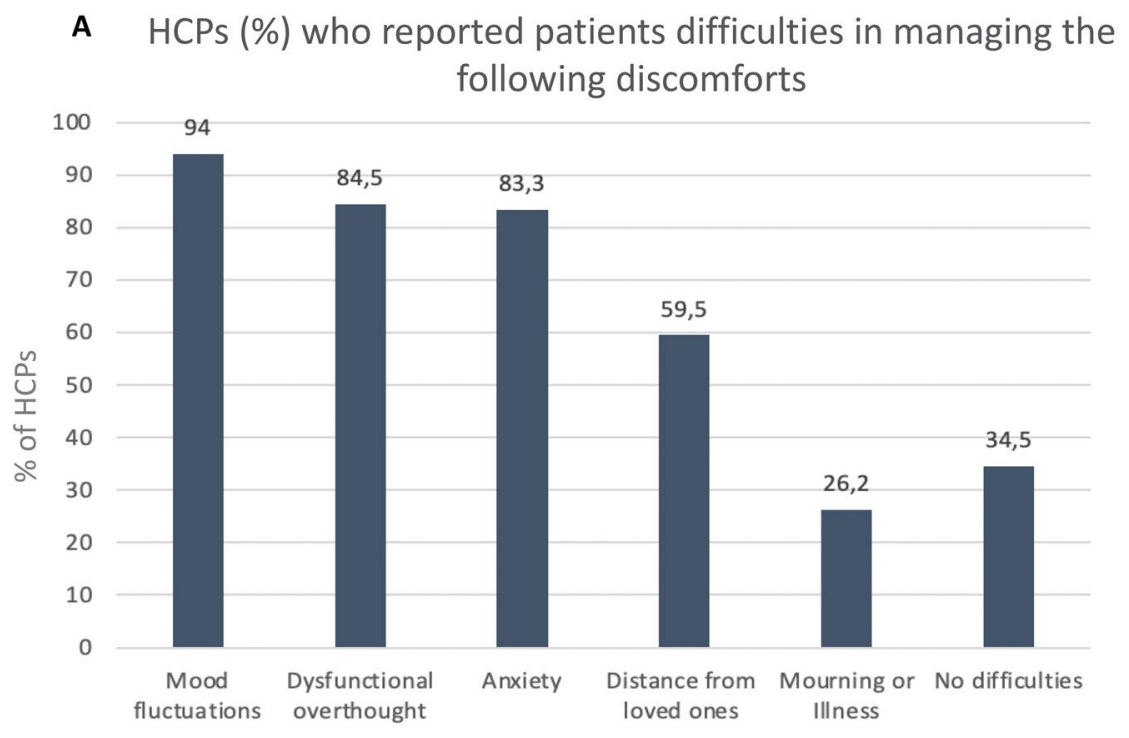

\section{B Difficulties experienced by HCPs during the online or in person meeting with their patients (\% of HCPs)}

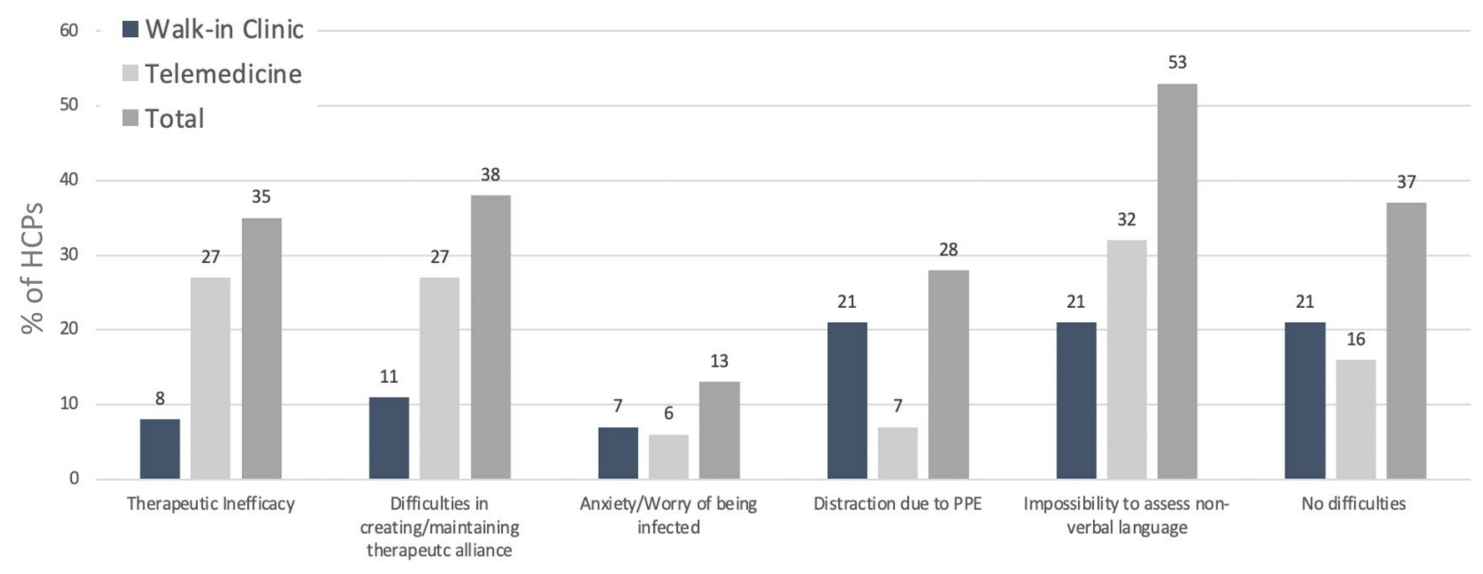

Fig. 2 Difficulties among HCPs and patients with eating disorder during the lockdown due to COVID-19 pandemic. a Difficulties experienced by patients according to HCPs (reported as HCPs \%). b Dif- ficulties experienced by HCPs during the online (telemedicine) or in person visits. HCPs Health Care Providers 


\section{Did you identify useful strategies to contain stress or improve ED symptoms?}

Among the effective strategies adopted during the lockdown, $14,3 \%$ of HCPs $(n=17)$ reported techniques of concentration and mindfulness. Such data is coherent with the listed difficulties: anxiety due to the possibility of being infected and the presence of PPE are all important distractors. The development of concentration techniques was thus considered essential for both, therapeutic success and anxiety management. For such purpose, respiration and relaxation exercises, group or individual online classes of yoga and meditation were considered effective. Furthermore, the creation of a routine planned with HCPs was also indicated as successful in some cases. Certain HCPs also suggested that an increase in the frequency of communication with their patients was able to contain their anxiety. However, a significant number of HCPs $(28,5 \%)$ did not identify any effective strategy to counteract patients' discomforts, an important data that further emphasize the relevance of our findings, potentially useful for this subgroup of therapists.

\section{Did the lockdown change in anyway your therapeutic intervention?}

According to HCPs, the main changes were related to the modification of the therapeutic setting and $12 \%$ of them emphasized that the difficulties they experienced were due to such alteration. However, $20 \%$ of HCPs stated they did not change in anyway their therapeutic approach.

According to some HCPs, the difficulties related to the online meetings were mainly due to the initial reorganization of the setting and were then resolved during the later visits. The lack of concentration of both, therapists and patients, was reported to be the main negative aspect of telemedicine. Consistently, $26 \%$ of HCPs stated it was the first time they tried such approach and the novelty was cause of distraction. Furthermore, some therapists also complained elevated tiredness related to such therapeutic modality. This method was in fact inevitably linked to an increased amount of time spent in the therapeutic assistance, due to the difficulties in managing such a novel approach. Another disadvantage of telemedicine was due to the impossibility to perform experiential activities, group classes and to assess patients' body weight. On the other side, the main modification during the in-person meetings in the walk-in clinic were related to the use of PPE, the need of keeping distances with patients and the requirement to disinfect the whole setting after each visit. According to therapists such changes, together with the fear of being infected, represented a great stressor during the therapeutic intervention. Furthermore, it is also important to emphasize that, in numerous cases telemedicine was considered challenging considering that it is based on patients' independence and auto-monitoring, all abilities very often compromised in patients with ED.

Surprisingly, 13 HCPs $(15,3 \%)$ commented positively the telemedicine experience. In such context in fact, HCPs were less scared of being infected and did not use PPE which often caused distractions during the assistance. Furthermore, the possibility to better organize their own free time and easily apply distancing rules made it the favorite strategy for several HCPs. Part of HCPs reported improvements in the relation with their patient due to the increased frequency of their communication and the possibility to foster patients' autonomy. One of the documented advantages of telemedicine is in fact the possibility to ascribe patients' achievements to themselves, being it less bound to a specific therapeutic environment [10]. Such phenomenon has the potential to boost patients' empowerment skills, cornerstone of ED therapy. Furthermore, since the online therapy is less time consuming compared to the in-person meeting (no need of commuting), the frequency of the encounters could be easily implemented and reduce the risk of drop-out. Telemedicine provides the advantage to easily reach patients that would not be otherwise treated for the lack of specialized centers in their own territory.

Our study has several limitations: we used a self-administered questionnaire formulated ad hoc due to the lack of validated questionnaires on the investigated topic; the cross-sectional nature of the investigation did not allow us to establish a cause-effect relation between lockdown and changes in therapeutic alliance or dysfunctional behaviors; due to the target of our questionnaire (HCPs as opposed to patients), we could not assess whether individuals experiencing variation in the frequency of bingeing conducts (e.g., those with $\mathrm{BN}$ who increased) were the same ones who switched to another disease (e.g., BN to AN); the relatively low sample size evaluated, especially for certain professional categories (e.g., educators).

\section{Conclusions}

Our is the first study investigating the experience of HCPs treating ED during the lockdown phase I in Italy. According to our survey, HCPs experienced a sense of therapeutic inefficiency and compromised therapeutic alliance. Besides personal difficulties due to the pandemic, HCPs reported to struggle in managing ED patients who experienced higher anxiety levels; social restrictions were in fact associated with changes in the frequency of dysfunctional behaviors among ED patients, especially among those suffering from BN. However, a peculiar case is represented by individuals with BED whose variation in the frequency of bingeing conducts was characterized by a comparable number of patients experiencing improvements and worsening. This 
finding deserves further investigation, since it suggests that a subgroup of BED subjects may benefit from a more controlled environment shared with the whole family. According to HCPs, effective strategies to contain stress consisted of the development of skills of concentration, through mindfulness, yoga, relaxation and respiration exercises. Studies with a longer duration and bigger sample size are needed to confirm our data, to assess the long-term effects of the lockdown on patients with ED and their HCPs and to identify useful strategies to resolve such potential negative outcomes.

\section{What is already known on this subject?}

The COVID-19 pandemic has caused psychological distress and increased frequency of dysfunctional behaviors among ED patients. None of the studies investigated the experience of HCPs treating ED.

\section{What this study adds?}

Knowledge of the impact of the Italian lockdown due to COVID-19 pandemic on HCPs treating ED and ED patients. Identification of useful strategies to counteract the pandemic-related anxiety.

Supplementary Information The online version contains supplementary material available at https://doi.org/10.1007/s40519-021-01116-5.

Acknowledgements Associazione "Mi Fido di Te" Onlus, Perugia, Italy.

Funding This study does not have any funding.

\section{Compliance with ethical standards}

Conflict of interest Authors have nothing to disclose.

Ethical approval This study conformed to the ethical principles of the Declaration of Helsinki for studies involving human subjects. Survey completion was anonymous; researchers do not possess any personal health information concerning any of the participants. Considering the nature of the survey (lack of any identifiable information).

Informed consent No signed informed consent was obtained from the responders.

\section{References}

1. Brooks SK, Webster RK, Smith LE et al (2020) The psychological impact of quarantine and how to reduce it: rapid review of the evidence. Lancet 395(10227):912-920. https://doi.org/10.1016/ S0140-6736(20)30460-8

2. Todisco P, Donini LM (2020) Eating disorders and obesity (ED\&O) in the COVID-19 storm. Eat Weight Disord. https://doi. org/10.1007/s40519-020-00938-Z

3. Haddad C, Zakhour M, Bou Kheir M et al (2020) Association between eating behavior and quarantine/confinement stressors during the coronavirus disease 2019 outbreak. J Eat Disord 8:40. https://doi.org/10.1186/s40337-020-00317-0

4. Termorshuizen JD, Watson HJ, Thornton LM et al (2020) Early impact of COVID-19 on individuals with self-reported eating disorders: a survey of $\sim 1,000$ individuals in the US and the Netherlands. Int J Eat Disord. https://doi.org/10.1002/eat.23353

5. Mukhtar S (2020) Psychological health during the coronavirus disease 2019 pandemic outbreak. Int J Soc Psychiatry 66(5):512516. https://doi.org/10.1177/0020764020925835

6. Phillipou A, Meyer D, Neill E et al (2020) Eating and exercise behaviors in eating disorders and the general population during the COVID-19 pandemic in Australia: initial results from the COLLATE project. Int J Eat Disord 53(7):1158-1165. https:// doi.org/10.1002/eat.23317

7. Weissman RS, Bauer S, Thomas JJ (2020) Access to evidencebased care for eating disorders during the COVID-19 crisis. Int $\mathrm{J}$ Eat Disord 53(5):369-376. https://doi.org/10.1002/eat.23279

8. Regier DA, Kuhl EA, Kupfer DJ (2013) The DSM-5: classification and criteria changes. World Psychiatry 12(2):92-98. https://doi. org/10.1002/wps.20050

9. Rodgers RF, Lombardo C, Cerolini S et al (2020) The impact of the COVID-19 pandemic on eating disorder risk and symptoms. Int J Eat Disord 53(7):1166-1170. https://doi.org/10.1002/ eat. 23318

10. Murphy C, Cooper Z, Dalle Grave R (2020) Challenges and opportunities for enhanced cognitive behaviour therapy (CBT-E) in light of COVID-19. Cognitiv Behav Therap 13:1-18. https:// doi.org/10.1017/S1754470X20000161

Publisher's Note Springer Nature remains neutral with regard to jurisdictional claims in published maps and institutional affiliations. 